\title{
The function of the glutamate-nitric oxide-cGMP pathway in brain in vivo and learning ability decrease in parallel in mature compared with young rats
}

\author{
Blanca Piedrafita, ${ }^{1}$ Omar Cauli, ${ }^{1}$ Carmina Montoliu, ${ }^{2}$ and Vicente Felipo ${ }^{1,3}$ \\ ${ }^{1}$ Laboratory of Neurobiology, Centro de Investigación Príncipe Felipe, Valencia 46013, Spain; ${ }^{2}$ Servicio de Hepatología, Hospital \\ Clinico Universitario, Departamento de Medicina, Universidad de Valencia, Valencia 46010 Spain
}

\begin{abstract}
Aging is associated with cognitive impairment, but the underlying mechanisms remain unclear. We have recently reported that the ability of rats to learn a Y-maze conditional discrimination task depends on the function of the glutamate-nitric oxide-cGMP pathway in brain. The aims of the present work were to assess whether the ability of rats to learn this task decreases with age and whether this reduction is associated with a decreased function of the glutamate-nitric oxide-cGMP pathway in brain in vivo, as analyzed by microdialysis in freely moving rats. We show that 7-mo-old rats need significantly more $(192 \pm 64 \%)$ trials than do 3-mo-old rats to learn the Y-maze task. Moreover, the function of the glutamate-nitric oxide-cGMP pathway is reduced by $60 \pm 23 \%$ in 7 -mo-old rats compared with 3-mo-old rats. The results reported support the idea that the reduction in the ability to learn the Y-maze task (and likely other types of learning) of mature compared with young rats would be a consequence of reduced function of the glutamate-nitric oxide-cGMP pathway.
\end{abstract}

Aging is associated with cognitive impairment, but the underlying mechanisms remain unclear. The molecular bases for different types of learning are not well known. NMDA receptors play a crucial role in some types of learning. Activation of NMDA receptors leads to increased calcium in the post-synaptic neuron. Calcium binds to calmodulin and activates neuronal nitric oxide synthase (nNOS) in those neurons, which express both the NMDA receptors and this enzyme, increasing nitric oxide (NO). $\mathrm{NO}$, in turn, activates soluble guanylate cyclase, increasing cGMP. Part of this cGMP is released to the extracellular fluid through cGMP transporters present in the cell membrane (Sager 2004).

Several reports indicate that activation of this glutamateNO-cGMP pathway is involved in some forms of learning (Danysz et al. 1995; Chen et al. 1997; Meyer et al. 1998). NO seems to mediate at least part of the role of NMDA receptors in learning. This is supported by studies showing that inhibition of NO synthase reduces learning of some spatial tasks (Ingram et al. 1998a,b; Zou et al. 1998)

The role of NO in learning may be mediated by its activation of soluble guanylate cyclase and the increase in cGMP. Some reports indicate that soluble guanylate cyclase and cGMP are important in learning and memory. Bernabeu et al. (1996, 1997) showed that, in rats, passive avoidance learning was associated with a time-dependent, learning-specific increase in cGMP (Bernabeu et al. 1996) and in cGMP-dependent protein kinase activity in hippocampus (Bernabeu et al. 1997)

The same group also showed that administration of a membrane permeable analog of cGMP facilitated memory consolidation (Bernabeu et al. 1996), while bilateral intrahippocampal administration of an inhibitor of soluble guanylate cyclase caused full amnesia for inhibitory avoidance learning when given im-

\section{${ }^{3}$ Corresponding author.}

E-mail vfelipo@cipf.es; fax 34-96-3289701.

Article is online at http://www.learnmem.org/cgi/doi/10.1101//m.541307. mediately after training (Bernabeu et al. 1997). These data indicate that the increase in cGMP produced by guanylate cyclase in response to NO has a crucial role in some types of learning.

We have recently shown that both the function of the glutamate-NO-cGMP pathway in cerebellum (Monfort et al. 2001) and the ability of rats to learn a Y-maze conditional discrimination task are reduced in rats with chronic liver failure or hyperammonemia and that learning ability may be restored by pharmacological manipulation of cGMP levels in brain (see Discussion).

The above studies suggest that the function of the glutamate-NO-cGMP pathway in brain and the concomitant increase in cGMP modulate the ability of rats to learn a Y-maze conditional discrimination task and likely other types of learning. If this is the case, impairment in the function of the glutamateNO-cGMP pathway could contribute to the decline in cognitive function associated to aging.

The aims of the present work were to assess whether the ability of rats to learn this Y-maze task decreases with age and whether this reduction is associated with a parallel impairment in the function of the glutamate-NO-cGMP pathway in brain in vivo, as analyzed by microdialysis in freely moving rats.

\section{Results}

We first tested whether the ability of rats to learn the Y-maze task is different in mature (7-mo-old) rats than in young (3-mo-old) rats. As shown in Figure 1, learning ability was significantly $(P<0.001)$ lower in 7 -mo-old male rats than in 3-mo-old male rats. At 3 mo of age, male rats need $52 \pm 3$ trials to learn the task, while at 7 mo of age, they need $97 \pm 10$ trials.

Similar results were obtained with female rats (Fig. 1). At 3 mo of age, female rats need $58 \pm 8$ trials to learn the task, while at 7 mo of age, they need $115 \pm 18$ trials.

As the results for males or females are not different, we also present in Figure 1 the pooled data from males and females. At 3 


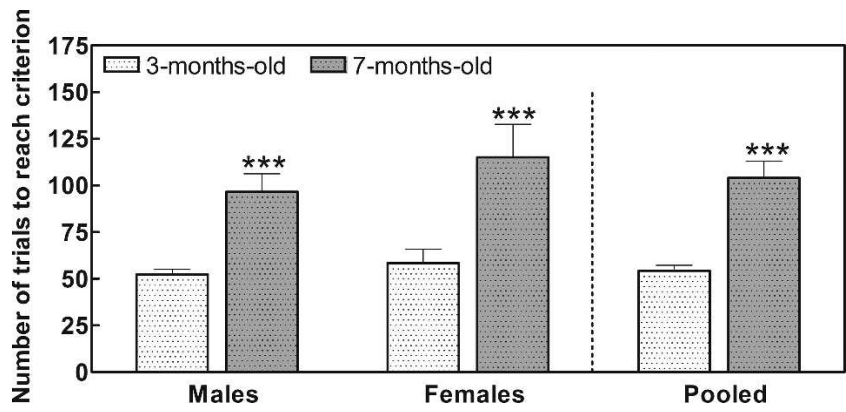

Figure 1. The ability to learn the Y-maze conditional discrimination task is lower in mature rats than in young rats; 3-mo-old and 7-mo-old male and female rats were subjected to the conditional discrimination learning test in the Y-maze as described in Materials and Methods. Values are the mean \pm SEM from seven rats per group and are given as the number of trials needed to learn. Data of different sex and age were analyzed using two-way ANOVA. There were no significant differences between sexes, but the effect of age is significant $(P<0.001)$. Values that are significantly different from young (3-mo-old) rats are indicated by asterisks: ${ }^{* *} p<0.001$ (two-way ANOVA). Pooled data were analyzed using Student's $t$-test to compare performance of young and mature rats. Significant difference from young ( 3 -mo-old) rats is indicated by asterisks: ${ }^{* * *} P<0.001$.

mo of age, the rats (males and females) need $54 \pm 3$ trials to learn the task, while at 7 mo of age they need $104 \pm 9$ trials. These data indicate that 7 -mo-old rats need $92 \%$ more trials than do 3 -moold rats to learn the task.

In all cases, learning ability was significantly lower at 7 mo of age than at 3 mo of age. Two-way ANOVA analysis showed no significant differences between sexes but a very significant $(P<0.001)$ effect of age.

We then tested whether the function of the glutamate-NOcGMP pathway in brain in vivo is also different in mature rats than in young rats. Administration of NMDA through the microdialysis probe activates the glutamate-NO-cGMP pathway and increases the formation of cGMP and its release to the extracellular fluid. As shown in Figure 2A, in 3-mo-old male rats the addition of NMDA increased significantly $(P<0.001)$ extracellular cGMP to $882 \pm 120 \%$ of basal. However, in 7 -mo-old male rats, the increase was significantly $(P<0.05)$ lower, reaching only $397 \pm 180 \%$ of basal (Fig. 2A).

Similar results were obtained for female rats. In 3-mo-old female rats, the addition of NMDA increased significantly $(P<0.001)$ extracellular cGMP to $864 \pm 70 \%$ of basal. However, in 7 -mo-old female rats, the increase was significantly $(P<0.01)$ lower, reaching only $272 \pm 152 \%$ of basal (Fig. 2B).

As the results for males or females are similar, we also present in Figure $2 \mathrm{C}$ the pooled data from males and females. In 3 -mo-old rats, the addition of NMDA increased significantly $(P<0.001)$ extracellular cGMP to $871 \pm 62 \%$ of basal. However, in 7 -mo-old rats, the increase was significantly $(P<0.001)$ lower, reaching only $343 \pm 198 \%$ of basal (Fig. 2 C).

These data indicate that the function of the pathway is reduced by $60 \pm 23 \%$ in 7 -mo-old rats compared with 3-mo-old rats.

The basal levels of extracellular cGMP were also significantly reduced in 7-mo-old rats compared with 3-mo-old rats. As shown in Figure 3, in males extracellular cGMP was $498 \pm 59 \mathrm{pM}$ at 3 mo and was significantly $(P<0.001)$ reduced (by 69\%) to $154 \pm 35 \mathrm{pM}$ at $7 \mathrm{mo}$ of age. Similar results were obtained for females. Extracellular cGMP was $513 \pm 103 \mathrm{pM}$ at 3 mo and was significantly $(P<0.01)$ reduced (by $65 \%)$ to $181 \pm 29 \mathrm{pM}$ at $7 \mathrm{mo}$ of age. As the results for males or females are similar, we also present in Figure 3 the pooled data from males and females. Extracellular cGMP was $505 \pm 197 \mathrm{pM}$ at $3 \mathrm{mo}$ and was significantly $(P<0.01)$ reduced (by $67 \%)$ to $166 \pm 91 \mathrm{pM}$ at 7 mo of age. Two-way ANOVA analysis showed no significant differences between sexes but a very significant $(P<0.001)$ effect of age.

We also analyzed whether the amount of NMDA receptors is different in the cerebellum of mature and young rats by quantifying by immunoblotting the content of the essential subunit NR1. As shown in Figure 4, the amount of the NR1 subunit in males at 7 mo was only $54 \pm 6 \%$ of the content at 3 mo of age $(P=0.01)$. In females the amount of NR1 was also lower $(80 \pm 15 \%)$ at 7 mo than at $3 \mathrm{mo}$, but the difference did not reach statistical significance. When the data of males and females were pooled, the amount of NR1 at 7 mo was significantly $(P=0.03)$ lower $(70 \pm 10 \%)$ than was the content at 3 mo (Fig. 4$)$.

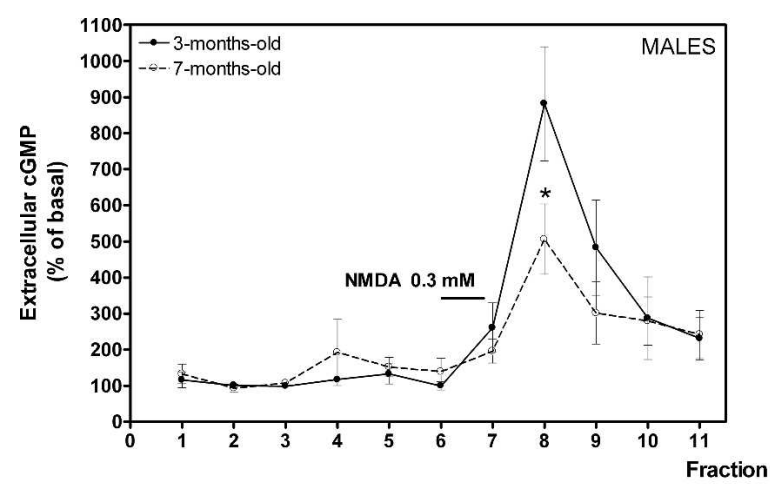

A

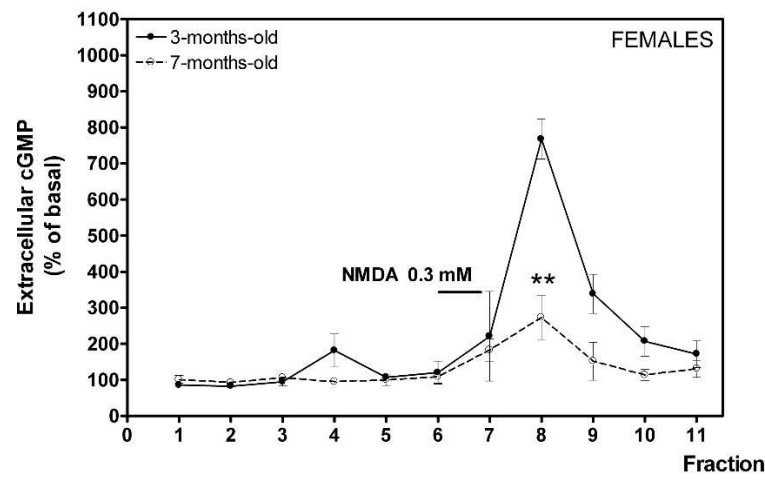

B

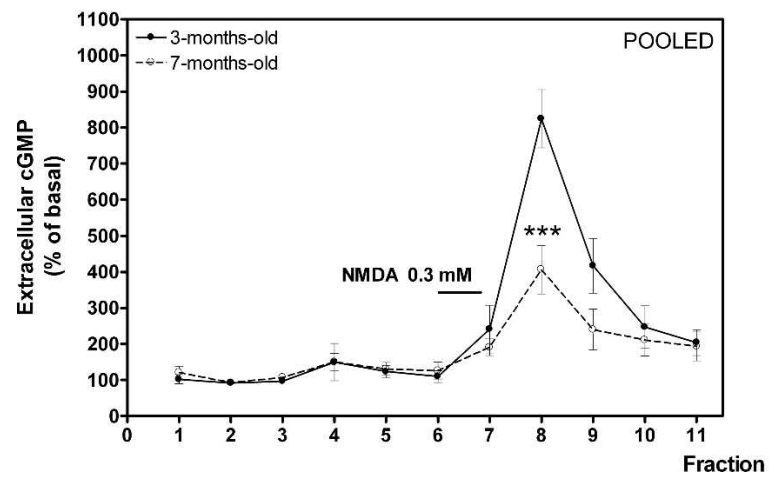

Figure 2. The function of the glutamate-NO-cGMP pathway in rat cerebellum in vivo is reduced in mature rats compared with young rats. The function of the Glu-NO-cGMP pathway was assessed by in vivo brain microdialysis. NMDA $0.3 \mathrm{mM}$ was infused at the times indicated, and extracellular CGMP was determined. Data are presented as percentage of basal values. Values are the mean \pm SEM from seven rats per group: $(A)$ male rats, $(B)$ female rats, and $(C)$ pooled data of males and females. Values that are significantly different from young (3-mo-old) rats are indicated by asterisks: ${ }^{*} P<0.05,{ }^{* *} P<0.01,{ }^{* * *} P<0.001$ (Student's $t$ test). 


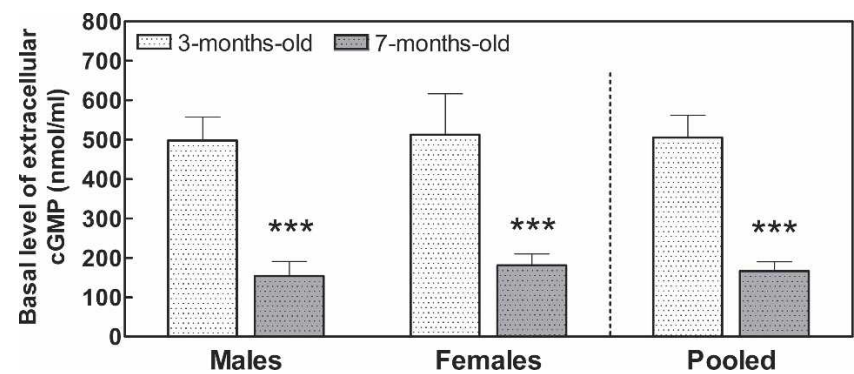

Figure 3. The basal concentration of extracellular cGMP in rat cerebellum is lower in mature rats than in young rats. The basal level of extracellular cGMP was measured in brain microdialysis samples (fractions 1-4 of Fig. 2). Values are mean \pm SEM from seven rats per group. Data of different sex and age were analyzed using two-way ANOVA. There were no significant differences between sexes, but the effect of age is significant $(P<0.001)$. Values that are significantly different from young (3mo-old) rats are indicated by asterisks: ${ }^{* *} P<0.01$, ${ }^{* * *} P<0.001$ (two-way ANOVA). Pooled data were analyzed using Student's $t$-test. Significant difference from young (3-mo-old) rats is indicated by asterisks: $* * * P<0.001$.

\section{Discussion}

The results reported show that in mature rats the function of the glutamate-NO-cGMP pathway in brain in vivo in freely moving rats and the ability of the rats to learn a Y-maze conditional discrimination task are reduced compared with young rats. Although parallel changes do not prove a cause-effect relationship, taking into account previous studies (see below), we believe that the decrease in the ability to learn this task in older rats is a consequence of the reduction in the function of the pathway. This is further supported by the fact that there is a significant $(P=0.0013, r=-0.769)$ correlation between the function of the glutamate-NO-cGMP pathway and the number of trials required by the rats to learn the Y-maze task (Fig. 5). A higher number of trials means a lower learning ability. This means that there is a direct correlation between the function of the pathway and learning ability: A higher function of the pathway is associated with higher learning ability.

We also show that the basal concentration of cGMP is also reduced in the extracellular fluid in the cerebellum of mature rats compared with young rats. Moreover, the decreases in basal extracellular cGMP and in the function of the glutamate-NOcGMP pathway are in the same order (60\%-70\%). This suggests that extracellular cGMP would depend on or reflect, at least in the cerebellum, the function of the pathway.

The function of the glutamate-NO-cGMP pathway is reduced in the cerebellum of rats with chronic hyperammonemia without liver failure (Hermenegildo et al. 1998). These rats show reduced ability to learn a conditional discrimination task in a Y-maze (Aguilar et al. 2000). The ability of these rats to learn the Y-maze task may be restored by increasing extracellular cGMP in brain by intracerebral administration of zaprinast, an inhibitor of the phosphodiesterase that degrades cGMP (Erceg et al. 2005a) or by oral administration of sildenafil, another inhibitor of the phosphodiesterase (Erceg et al. 2005b).

The function of the glutamate-NO-cGMP pathway is also reduced in the cerebellum of rats with chronic liver failure induced by porta-caval anastomosis (Monfort et al. 2001). These rats also show reduced ability to learn a conditional discrimination task in the Y-maze, which may be also restored by increasing extracellular cGMP in the cerebellum by oral administration of sildenafil (Erceg et al. 2005b).

These studies strongly support the idea that the ability to learn the Y-maze conditional discrimination task depends on the function of the glutamate-NO-cGMP pathway in the brain. The reduction in the function of the pathway in mature compared with young rats would be therefore responsible for the decrease in the ability to learn this task in 7-mo-old rats compared with 3-mo-old rats.

The glutamate-NO-cGMP pathway shows similar patterns of activation in the hippocampus, striatum, and cerebellum (Fedele and Raiteri 1999; Pepicelli et al. 2004). Also, in an acute paradigm, ammonia activates the pathway similarly in the cerebellum and striatum (Hermenegildo et al. 2000; Hilgier et al. 2003). This study was performed in the cerebellum because the expression of the pathway is high in this area, allowing more accurate quantitative determinations, which are more difficult to obtain in the hippocampus (considered the main memory structure of the brain) because it has lower basal levels of extracellular cGMP. The changes in the function of the pathway and in extracellular cGMP in the cerebellum may be considered a marker of the effects of age in other brain areas, including the hippocampus and striatum. Moreover, it should be noted that recent studies show that the cerebellum also plays a role in spatial learning that is not the result of motor components but of cognitive components of spatial learning (Martin et al. 2003). A direct contribution of the glutamate-NO-cGMP pathway in the cerebellum to modulation of learning therefore cannot be excluded.

The mechanisms by which the function of the glutamate$\mathrm{NO}-\mathrm{cGMP}$ pathway is lower in mature rats than in young rats are
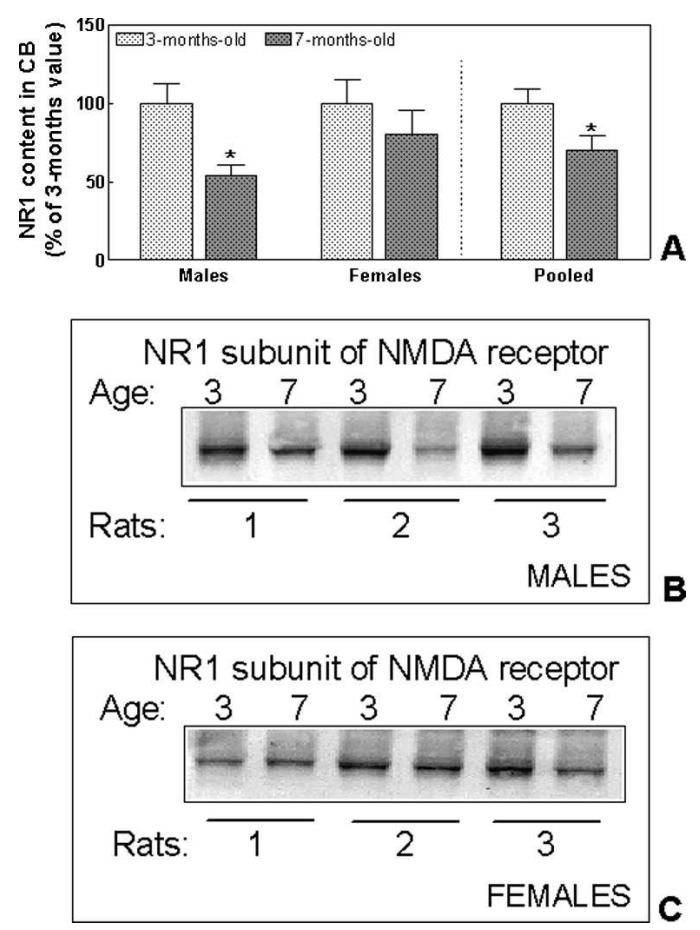

Figure 4. The amount of NMDA receptors in cerebellum is lower in mature male rats than in young male rats. Thirty micrograms of protein from homogenates of the cerebellum from 3-mo-old or 7-mo-old rats was subjected to SDS-polyacrylamide gel electrophoresis using an $8 \%$ acrylamide gel. The NR1 subunit was visualized by immunoblotting as indicated in Materials and Methods. Typical immunoblotting is shown for males $(B)$ and females $(C)$. The intensities of the bands were quantified and are shown in $A$. Values are the mean \pm SEM of five rats per group and are expressed as percentage of NR1 amount in 3-mo-old rats. Data of different sex and age were analyzed using two-way ANOVA. There were no significant differences between sexes, but the effect of age is significant $(P<0.05)$. Values that are significantly different from young (3-mo-old) rats are indicated by asterisks: ${ }^{*} P<0.05$ (two-way ANOVA). Pooled data were analyzed using Student's $t$-test. Significant difference from young (3-mo-old) rats is indicated by asterisks: ${ }^{*} P<0.05$. 


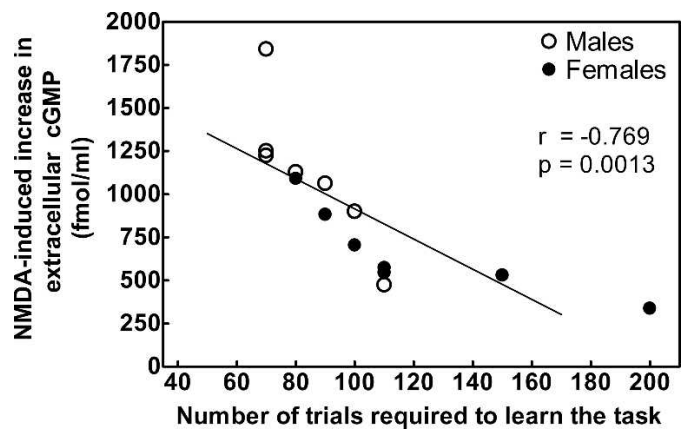

Figure 5. There is a good correlation between NMDA-induced increase in extracellular cGMP and the ability to learn the Y-maze task. Individual data for 7-mo-old males and females are plotted. The function of the glutamate-NO-cGMP pathway is expressed as the increase in CGMP induced by administration of NMDA, i.e., the increase in CGMP in fractions 7-9 of Figure 2. There is a negative and significant $(r=-0.769$; $P=0.0013$ ) correlation between the function of the pathway and the number of trials required to learn the task. A higher number of trials means a lower learning ability. This means that there is a direct correlation between the function of the pathway and learning ability: A higher function of the pathway correlates with higher learning ability.

not completely clear by now. It is likely that a main contributor to this reduction would be a decrease in the amount of NMDA receptors. We show here that the amount of NMDA receptors is strongly reduced in males at 7 mo of age compared with 3 mo of age. This may explain, or at least would contribute to, the reduced function of the glutamate-NO-cGMP pathway.

However, the amount of NMDA receptors is only slightly, and not significantly, reduced in females at 7 mo of age compared with 3 mo of age, while the function of the pathway and learning is also impaired in females at $7 \mathrm{mo}$. This suggests that, in addition to the reduction in the amount of NMDA receptors, impairment of its function or in some other step of the pathway would also contribute to its reduced function and to the associated decrease in learning ability. Other steps that could be altered with age are activation of guanylate cyclase by NO or the function of cGMP transporters. Also changes in the spatial localization of the proteins of the pathway (calmodulin, nNOS, guanylate cyclase) could influence the efficiency of the function of the pathway. As far as we know, there are no reports indicating that these steps could change with age.

A reduction with age in the amount of NMDA receptors has been consistently found in most brain areas (hippocampus, striatum, frontal cortex, hypothalamus, etc.) in different studies. Similar results have been reported when the amount of NMDA receptors is determined by measuring binding of ligands or by immunoblotting (Magnusson and Cotman 1993; Castorina et al. 1994; Scheuer et al. 1995; Vernet et al. 1998; Mothet et al. 2006). A decrease in the amount of NMDA receptors would result in a reduced function of the associated signal transduction pathways, including the glutamate-NO-cGMP pathway.

Another main player in this pathway is nNOS. Total NO synthase activity increases with age in brain, but this has been attributed to an increase in the content of the inducible form of NOS (iNOS) (Siles et al. 2002; Liu et al. 2004). It seems that maturation or aging does not affect significantly the nNOS. Yu et al. (2000) reported that the amount of mRNA and of protein for nNOS is significantly reduced in cerebellum of 6-mo-old rats compared with 2-mo-old rats. However, the activity of nNOS was not different. Vernet et al. (1998) found no effect of aging on nNOS content in hypothalamus.

These studies and the data reported here support, therefore, that the reduced function of the glutamate-NO-cGMP pathway in 7-mo-olds compared with 3-mo-olds would be due, at least in part, to the reduced amount of NMDA receptors. In addition, impairment of its function or in some other step of the pathway would also contribute to the reduced function of the pathway which, in turn, would contribute to the age-associated decrease in learning ability.

\section{Materials and Methods}

\section{Rats}

Learning tests and microdialysis studies were performed in 3-moold and 7-mo-old Wistar rats. Both male and female rats were used and the results were analyzed separately to assess whether there is some gender-associated difference. All animal procedures were approved by the Institute and met the guidelines of the European Union for care and management of experimental animals.

\section{Y-maze learning test}

Learning ability was tested, as described by Murray and Ridley (1997), in a wooden Y-maze, which had three arms of equal size (60 $\mathrm{cm}$ long, $11.5 \mathrm{~cm}$ wide, and $25 \mathrm{~cm}$ high). The arm where the rats were placed at the beginning of each trial was considered the start arm. The other arms, each of which had a food cup located at the end, were considered the choice arms. Pre-training was carried out during $4 \mathrm{~d}$ with all rats to familiarize them with the maze. The whole area of the choice arms of the maze was covered by black or white inserts $(59 \mathrm{~cm}$ long, $11 \mathrm{~cm}$ wide, and $25 \mathrm{~cm}$ high). In each trial, rats were rewarded for choosing the left arm when the inserts were black and the right arm when the inserts were white. The reward for the correct response consisted of four food pellets placed in the food cup at the end of the correct arm. If the rat made an incorrect response, it was allowed to go to the empty food cup at the end of the incorrect arm and was removed after $5 \mathrm{sec}$. Rats were trained for 10 trials per day, with an intertrial interval of $\sim 5 \mathrm{~min}$ in their home cage, until the completion of a criterion of 10 correct responses in 10 consecutive trials or until a maximum of 250 trials.

\section{Analysis of the function of the glutamate-NO-cGMP pathway in brain in vivo by microdialysis in freely moving rats}

Rats were generally anesthetized by careful intraperitoneal injection of $40 \mathrm{mg} / \mathrm{kg}$ sodium pentobarbital (Euta Lender). The animal's head was placed in a stereotaxic frame (David Kopf Instruments), and a microdialysis guide (CMA/12 guide cannula, CMA/ Microdialysis $\mathrm{AB}$ ) was implanted in the cerebellum. Briefly, a medial incision was made over the skull, and a burr hole $(\varnothing=1$ $\mathrm{mm}$ ) was drilled to allow vertical access to the cerebellum. The coordinates were $10.2 \mathrm{~mm}$ posterior to and $1.2 \mathrm{~mm}$ to the right of the bregma, and $1 \mathrm{~mm}$ below the duramater surface, according to the method of Pellegrino et al. (1979) and the corrections of Whishaw et al. (1977) for rats $>320 \mathrm{~g}$. The guide was secured to the skull with miniature screws and dental cement, and the skin was sutured. Animals were allowed to recover for $24-48 \mathrm{~h}$ in their cages with free access to pellet food and water.

The day of the experiment, the animals were placed in the microdialysis bowl of the BAS system for freely moving animals (BAS BeeKeeper, Bioanalytical Systems), and a microdialysis probe (CMA/12; $3 \mathrm{~mm}$ long, $500 \mu \mathrm{m}$ O.D.) was implanted carefully in the freely moving animal. The probes were continually perfused with artificial cerebrospinal fluid (aCSF) at a flow rate of $3 \mu \mathrm{L} / \mathrm{min}$ using a BAS Baby Bee microperfusion pump. The composition of aCSF was: $\mathrm{NaCl} 145 \mathrm{mM}, \mathrm{KCl} 3.0 \mathrm{mM}, \mathrm{CaCl}_{2} 2.26$ $\mathrm{mM}$, buffered at $\mathrm{pH} 7.4$ with $2 \mathrm{mM}$ phosphate buffer (as described by Fedele et al. 1996), but we replaced $\mathrm{MgCl}_{2}$ with $\mathrm{CaCl}_{2}$ to avoid the blocking effect of $\mathrm{Mg}^{2+}$ on NMDA receptor, and filtered through $0.45-\mu \mathrm{m}$ pore-size Millipore filters. The probes were dialyzed continuously for $2-3 \mathrm{~h}$, because preliminary studies demonstrated extracellular cGMP was stabilized within this interval. After the stabilization period, consecutive samples were collected every 20 min with a fraction collector, covered, and 
stored at $-20^{\circ} \mathrm{C}$ until the analysis of cyclic GMP. We have previously shown (Hermenegildo et al. 1998) that the increase in extracellular cGMP induced by NMDA is linear between 0.1 and 0.5 $\mathrm{mM}$. So that, to activate the glutamate-NO-cGMP pathway, we added $0.3 \mathrm{mM}$ NMDA at the times indicated in the legend to Figure 2. cGMP was determined using the BIOTRAK cGMP enzyme immunoassay kit from Amersham.

\section{Determination of the amount of NMDA receptors by immunoblotting}

Rats were sacrificed at 3 or 7 mo of age: The cerebellum was dissected out and immediately freeze-clamped in liquid nitrogen and then stored at $-80^{\circ} \mathrm{C}$. Whole cerebella were homogenized in 5 volumes of a buffer containing Tris- $\mathrm{HCl} 50 \mathrm{mM}(\mathrm{pH} 7.5) ; \mathrm{NaCl}$ $50 \mathrm{mM}$, EGTA $10 \mathrm{mM}$, EDTA $5 \mathrm{mM}$, pNPP $4 \mathrm{mM}$, sodium pyrophosphate $2 \mathrm{mM}$, sodium orthovanadate $1 \mathrm{mM}$, NaF $1 \mathrm{mM}$, leupeptin $20 \mu \mathrm{g} / \mathrm{mL}$, aprotinin $20 \mu \mathrm{g} / \mathrm{mL}$, and PMSF $1 \mathrm{mM}$ and sonicated. Protein concentration was determined by the bicinchonic acid method (Pierce). Samples were subjected to SDSpolyacrylamide gel electrophoresis and immunoblotting using monoclonal antibodies against the NR1 subunit of the NMDA receptor (Pharmingen, BD Biosciences) Secondary antibodies conjugated with alkaline phosphatase were from Sigma. After development, images were captured with a Hewlett Packard Scanjet $5300 \mathrm{C}$. The intensities of the bands were measured using the program AlphaImager 2200 (AlphaEase FC Software 3.1.2 from Alpha Innotech corporation).

\section{Statistical analysis}

Learning test and cGMP levels data were analyzed using two-way ANOVA followed by Bonferroni post-hoc with age and sex as variables. In the case of pooled data, unpaired Student's $t$-tests were used. $P$ values $<0.05$ were considered statistically significant. All values were given as mean $\pm \mathrm{SE}$. All statistical analyses were made using the GraphPad Prism 4.0 program.

\section{Acknowledgments}

This work was supported by grants from the European Commission. Program Food Quality and Safety. FOOD-CT-2003-506143 and from Ministerio de Educación y Ciencia (SAF2002-00851, SAF2004-0001-E and SAF2005-06089) and from Ministerio de Sanidad (Red G03-155 and FIS PI050253) of Spain and by grants from Consellería de Empresa, Universidad y Ciencia (Grupos03/ 001, GV04B-055, GV04B-012, GVS05/082, ACOMP06/005) and AP005/06 from Conselleria de Sanitat of Generalitat Valenciana.

\section{References}

Aguilar, M.A., Minarro, J., and Felipo, V. 2000. Chronic moderate hyperammonemia impairs active and passive avoidance behavior and conditional discrimination learning in rats. Exp. Neurol. 161: 704-713.

Bernabeu, R., Schmitz, P., Faillace, M.P., Izquierdo, I., and Medina, J.H. 1996. Hippocampal cGMP and cAMP are differentially involved in memory processing of inhibitory avoidance learning. Neuroreport 7: 585-588.

Bernabeu, R., Schroder, N., Quevedo, J., Cammarota, M., Izquierdo, I., and Medina, J.H. 1997. Further evidence for the involvement of a hippocampal cGMP/cGMP-dependent protein kinase cascade in memory consolidation. Neuroreport 8: 2221-2224.

Castorina, M., Ambrosini, A.M., Pacific, L., Ramacci, M.T., and Angelucci, L. 1994. Age-dependent loss of NMDA receptors in hippocampus, striatum, and frontal cortex of the rat: prevention by acetyl-L-carnitine. Neurochem. Res. 19: 795-798.

Chen, J., Zhang, S., Zuo, P., and Tang, L. 1997. Memory-related changes of nitric oxide synthase activity and nitrite level in rat brain. Neuroreport 8: 1771-1774.

Danysz, W., Zajaczkowski, W., and Parsons, C.G. 1995. Modulation of learning processes by ionotropic glutamate receptor ligands. Behav. Pharmacol. 6: 455-474.

Erceg, S., Monfort, P., Hernandez-Viadel, M., Llansola, M., Montoliu, C., and Felipo, V. 2005a. Restoration of learning ability in hyperammonemic rats by increasing extracellular cGMP in brain. Brain Res. 1036: 115-121.

Erceg, S., Monfort, P., Hernandez-Viadel, M., Rodrigo, R., Montoliu, C., and Felipo, V. 2005b. Oral administration of sildenafil restores learning ability in rats with hyperammonemia and with portacaval shunts. Hepatology 41: 299-306.

Fedele, E. and Raiteri, M. 1999. In vivo studies of the cerebral glutamate receptor/NO/cGMP pathway. Prog. Neurobiol. 58: 89-120.

Fedele, E., Jin, Y., Varnier, G., and Raiteri, M. 1996. In vivo microdialysis study of a specific inhibitor of soluble guanylyl cyclase on the glutamate receptor/nitric oxide/cyclic GMP pathway. Br. J. Pharmacol. 119: 590-594.

Hermenegildo, C., Montoliu, C., Llansola, M., Munoz, M.D., Gaztelu, J.M., Minana, M.D., and Felipo, V. 1998. Chronic hyperammonemia impairs the glutamate-nitric oxide-cyclic GMP pathway in cerebellar neurons in culture and in the rat in vivo. Eur. J. Neurosci. 10: 3201-3209.

Hermenegildo, C., Monfort, P., and Felipo, V. 2000. Activation of NMDA receptors in rat brain in vivo following acute ammonia intoxication. Characterization by in vivo brain microdialysis. Hepatology 31: 709-715.

Hilgier, W., Anderzhanova, E., Oja, S.S., Saransaari, P., and Albrecht, J. 2003. Taurine reduces ammonia- and N-methyl-D-aspartate-induced accumulation of cyclic GMP and hydroxyl radicals in microdialysates of the rat striatum. Eur. J. Pharmacol. 468: 21-25.

Ingram, D.K., Spangler, E.L., Kametani, H., Meyer, R.C., and London, E.D. 1998a. Intracerebroventricular injection of $\mathrm{N} \omega$-nitro-L-arginine in rats impairs learning in a 14-unit T-maze. Eur. J. Pharmacol. 341: $11-16$.

Ingram, D.K., Spangler, E.L., Meyer, R.C., and London, E.D. 1998 b. Learning in a 14-unit T-maze is impaired in rats following systemic treatment with Nw-nitro-L-arginine. Eur. J. Pharmacol. 341: 1-9.

Liu, P., Smith, P.F., Appleton, I., Darlington, C.L., and Bilkey, D.K. 2004 Age-related changes in nitric oxide synthase and arginase in the rat prefrontal cortex. Neurobiol. Aging 25: 547-552.

Magnusson, K.R. and Cotman, C.W. 1993. Effects of aging on NMDA and MK801 binding sites in mice. Brain Res. 604: 334-337.

Martin, L.A., Goldowitz, D., and Mittleman, G. 2003. The cerebellum and spatial ability: Dissection of motor and cognitive components with a mouse model system. Eur. J. Neurosci. 18: 2002-2010.

Meyer, R.C., Knox, J., Purwin, D.A., Spangler, E.L., and Ingram, D.K. 1998. Combined stimulation of the glycine and polyamine sites of the NMDA receptor attenuates NMDA blockade-induced learning deficits of rats in a 14-unit T-maze. Psychopharmacology 135: 290-295

Monfort, P., Corbalan, R., Martinez, L., Lopez-Talavera, J., Cordoba, J., and Felipo, V. 2001. Altered content and modulation of soluble guanylate cyclase in the cerebellum of rats with portacaval anastomosis. Neuroscience 104: 1119-1125.

Mothet, J.P., Rouaud, E., Sinet, P.M., Potier, B., Jouvenceau, A., Dutar, P., Videau, C., Epelbaum, J., and Billard, J.M. 2006. A critical role for the glial-derived neuromodulator D-serine in the age-related deficits of cellular mechanisms of learning and memory. Aging Cell 5: $267-274$.

Murray, T.K. and Ridley, R.M. 1997. The effect of dizocilpine (MK-801) on conditional discrimination learning in the rat. Behav. Pharmacol. 8: $383-388$.

Pellegrino, L.J., Pellegrino, A.S., and Cushman, A.J. 1979. A stereotaxic atlas of the rat brain. Plenum Press, New York.

Pepicelli, O., Raiteri, M., and Fedele, E. 2004. The NOS/sGC pathway in the rat central nervous system: A microdialysis overview. Neurochem. Int. 45: 787-797.

Sager, G. 2004. Cyclic GMP transporters. Neurochem. Int. 45: 865-873.

Scheuer, K., Stoll, S., Paschke, U., Weigel, R., and Muller, W.E. 1995. $\mathrm{N}$-methyl-D-aspartate receptor density and membrane fluidity as possible determinants of the decline of passive avoidance performance in aging. Pharmacol. Biochem. Behav. 50: 65-70.

Siles, E., Martinez-Lara, E., Canuelo, A., Sanchez, M., Hernandez, R., Lopez-Ramos, J.C., Del Moral, M.L., Esteban, F.J., Blanco, S., Pedrosa, J.A., et al. 2002. Age-related changes of the nitric oxide system in the rat brain. Brain Res. 956: 385-392.

Vernet, D., Bonavera, J.J., Swerdloff, R.S., Gonzalez-Cadavid, N.F., and Wang, C. 1998. Spontaneous expression of inducible nitric oxide synthase in the hypothalamus and other brain regions of aging rats. Endocrinology 139: 3254-3261.

Whishaw, I.Q., Cioe, J.D., Previsich, N., and Kolb, B. 1977. The variability of the interaural line vs the stability of bregma in rat stereotaxic surgery. Physiol. Behav. 19: 719-722.

Yu, W., Juang, S., Lee, J., Liu, T., and Cheng, J. 2000. Decrease of neuronal nitric oxide synthase in the cerebellum of aged rats. Neurosci. Lett. 291: 37-40.

Zou, L.B., Yamada, K., Tanaka, T., Kameyama, T., and Nabeshima, T. 1998. Nitric oxide synthase inhibitors impair reference memory formation in a radial arm maze task in rats. Neuropharmacology 37: 323-330.

Received January 23, 2007; accepted in revised form February 20, 2007. 


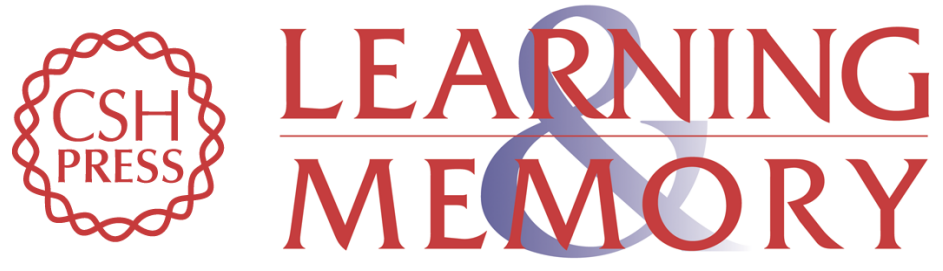

\section{The function of the glutamate-nitric oxide-cGMP pathway in brain in vivo and learning ability decrease in parallel in mature compared with young rats}

Blanca Piedrafita, Omar Cauli, Carmina Montoliu, et al.

Learn. Mem. 2007, 14:

Access the most recent version at doi:10.1101//m.541307

\section{License}

Email Alerting Receive free email alerts when new articles cite this article - sign up in the box at the Service top right corner of the article or click here. 